\title{
Les petites installations hydroélectriques dans les pays en voie de développement
}

\section{Small hydroelectric installations in the developing countries}

\author{
J. Francou
}

Électricité de France

Conseiller technique au Centre d'Ingénierie extérieure

\section{Electricité et développement}

On a parfois écrit que le développement économique d'une nation était lié à sa production d'électricité. Qu'en est-il au juste?

Avec les réserves d'usage concernant l'utilisation de statistiques établies à l'échelle du monde entier, on peut pointer sur un graphique (Fig. 1), pour une centaine de pays, le revenu (PNB) par tête, exprimé en Francs (1978) et la consommation par tête (en 1978) exprimée en $\mathrm{kWh}$.

Si l'on cherche à lier ces deux grandeurs, on constate que la relation doit être de la forme: $W=a R^{3 / 2}$.

Si l'on exprime $R$ en kilofrancs, on a "grosso-modo" $W=30 R^{3 / 2}$ pour les pays dont le PNB a dépassé, en 1978,25000 francs par tête, et $W=70 R^{3 / 2}$ pour les pays dont le revenu a été inférieur à ce chiffre.

Parmi les pays "riches" (en exceptant l'Arabie Saoudite et la Libye...) le graphique permet de constater que la France a été la plus économe en énergie électrique.

Il permet aussi de se rendre compte à quel point la "corrélation" est lâche, puisque l'on va de $W=12 R^{3 / 2}$ pour la Suisse à $W=60 R^{3 / 2}$ pour la Norvège.

Pour les pays moins riches, la corrélation est encore moins évidente, puisque l'on va de $W=15 R^{3 / 2}$ pour l'Albanie, l'Indonésie, l'Iran, ou le Nigéria, à $W=250 R^{3 / 2}$ pour la Corée du Nord, le Gabon ou la Zambie, pays dont la production est en totalité ou en majorité d'origine hydraulique.

L'analyse détaillée de ce graphique déborderait de notre sujet, mais on peut en retenir deux points qui paraissent importants :

- Les pays les plus déshérités sont séparés des pays très développés (Etats-Unis par exemple) par un coefficient 60 en ce qui concerne le revenu par tête, et par un coefficient 500 en ce qui concerne la consommation d'énergie électrique (on retrouve la puissance 3/2);
- Lorsqu'un pays est en développement effectif de $10 \%$ par an, on doit s'attendre à voir ses besoins en électricité s'accrô̂tre au taux de $15 \%$, ce qui conduit au doublement de la consommation en 5 ans. S'il ne peut être aujourd'hui que très rare d'envisager le développement d'une nation entière au taux de $10 \%$, il n'est pas exclu pour autant de trouver, sur des périmètres plus restreints, des villes ou des collectivités susceptibles d'un tel développement, à la condition qu'elles en aient les moyens. Et ce peut être le privilège d'aménagements hydrauliques de puissances modestes que de permettre de tels essors localisés.

\section{Le "Choc pétrolier"}

Il est bien connu que l'augmentation du prix des produits pétroliers est beaucoup plus grave encore pour les pays en développement que pour les pays développés.

Un exemple concret permettra de mesurer les répercussions de cette contrainte.

Dans le N.E. du Zaire, la ville d'Isiro est située au centre d'une région agricole au potentiel très important en café, coton, huile de palme, etc. Elle est située à plus de $2000 \mathrm{~km}$ à vol d'oiseau du port de Matadi, et l'énergie électrique y est produite par des groupes Diesel.

La production annuelle est donnée par le graphique (Fig. 2)

Après les vicissitudes d'ordre politique des années 1960 à 1965, la production rédémarre au rythme assez "classique" de + $7 \%$ par an. A partir de 1974, elle s'effondre pour rejoindre pratiquement, en 1980 , le niveau de 1960.

Il est très probable que disposant ainsi de $2 \mathrm{GWh}$ au lieu des 5,5 GWh qu'elle aurait dû normalement consommer, cette région doit connaitre un brusque recul de 15 ans dans son essor économique.

Et c'est donc dans des conditions économiques difficiles que l'on se préoccupe maintenant d'aménager 


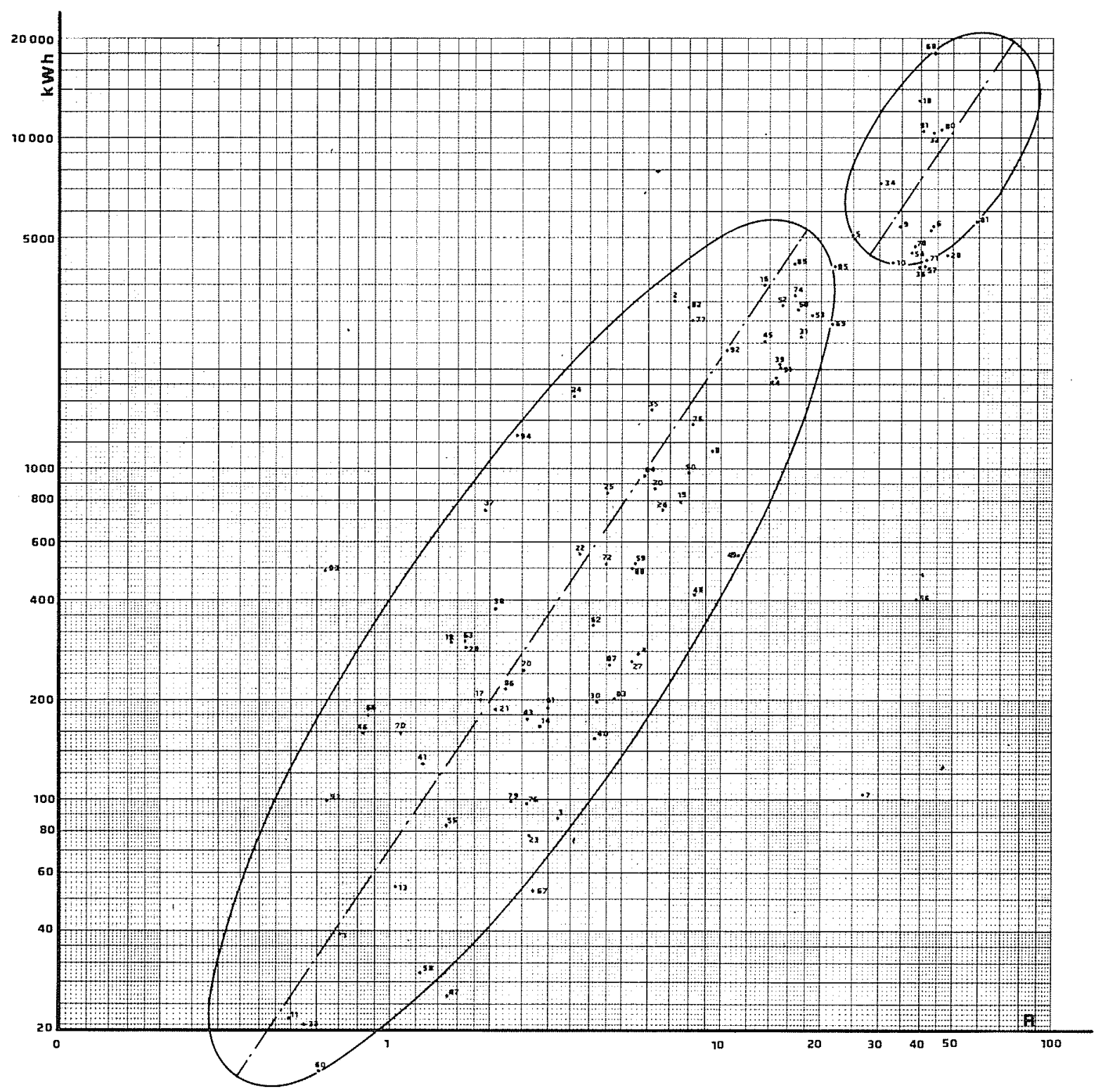

.4

\begin{tabular}{|c|c|c|c|c|c|c|c|c|c|c|c|c|c|c|c|c|}
\hline & & $E$ & $\mathrm{kth}$ & & & $\bar{F}$ & $k$ hn & & $\hat{F}$ & kwh & & $\mathrm{F}$ & xhoh & & $\mathrm{F}$ & kith \\
\hline 1 & Afghanistan & 713 & .39 & 21 & Chlne & 2120 & 191 & 41 Guinée & 1269 & 129 & 51. Maroc & 3015 & 192 & 81 Suisse & 60190 & 5560. \\
\hline 2 & Afrtque du Sud & 7271 & $\begin{array}{ll}3280 \\
-1\end{array}$ & 22 & Colombie & 3752 & 559 & 42 Haute-Volta & 600 & 10 & 62 Maurice & 4204 & 340 & 82 Surinam & 8000 & 3555 \\
\hline 3 & Albante & 3244 & 82 & 23 & Congo & 2639 & 69 & 43 Honduras & 2615 & 177 & 63 Murttanle & 1700 & 296 & 83 Syrie & 4847 & 204 \\
\hline 4 & Algérie & 5695 & 279 & 24 & Corée Nord & 3604 & \pm 682 & 44 Hong-Kong & 14415 & 1937 & 64 Mexique & 5960 & 836 & 84 Tchad & 666 & 18 \\
\hline 5 & Allem.Dém. & 25641 & 5230 & 25 & Corée Sud & 4528 & 864 & 45 Hongrie & 13800 & 2440 & 65 Hozamblque & 805 & 310 & 85 Tchécoslovaquie & 22200 & 4420. \\
\hline 6 & Allem.Féd & 43974 & $54 \infty$ & 26 & Costa-rica & 6753 & 773 & 46 Inde & 842 & 16.1 & 65 Niger & 864 & 185 & 86 Therlande & 2248 & 227 \\
\hline 7 & Arable Saoud. & 26200 & 105 & 27 & Côte d'Ivotre & 5437 & 272 & 47 Indonéste & 2484 & 27 & 57 Nigéria & 2700 & 53 & 87 Tunisie & 4612 & 263 \\
\hline 8 & Argentine & 9401 & $\begin{array}{lll}1 & 151\end{array}$ & 28 & Danemack & $48 \quad 330$ & 4550 & 48 Irak & 8400 & 420 & 68 Norvège & 44555 & 18500 & 88 Turquie & 5435 & 523 \\
\hline 9 & Australie & 34968 & 5520 & 29 & Egypte & 1704 & 310 & 49 Iran & 10672 & 554 & 69 Nouv.Zélande & 21880 & 2830 & 89 U.R.S.S. & 16220 & 4270 \\
\hline 10 & Autriche & 34707 & $\begin{array}{lll}4 & 480\end{array}$ & 30 & Equateur & 4233 & 198 & 50 Irlande & 16960 & 3072 & 70 Pakistan & 1095 & 159 & 90 uruguay & 8000 & 996 \\
\hline 11 & Bangladesh & 495 & 22 & 31 & Espagne & 17500 & 2510 & 51 Islande & 40900 & 11818 & 71 Pays-Bas & 41950 & 4250 & 91 Vénézuéla & 15300 & 2120 \\
\hline 12 & Belglque & 44150 & $46 \infty$ & 32 & Etats-unts & 43570 & 10520 & 52 Iśrä̈I & 15512 & 3185 & 72 Pérou & 4523 & 520 & 92 Yougoslavie & 10335 & 2360 \\
\hline 13 & Bentn & 1064 & 55 & 33 & Ethiople & 552 & 21 & 53 Italie & 18848 & 2940 & 73 Philippines & 2576 & 255 & 93 ZaIre & 663 & 493 \\
\hline 14 & Bolluie & 2857 & 168 & 34 & Pinl ande & 30.590 & 7470 & 54 Japon & $38 \quad 160$ & 4630 & 74 Pologne & 16830 & $\begin{array}{lll}3 & 303\end{array}$ & 94 Zamble & 2452 & 1320 \\
\hline 15 & Brésil & 7573 & 801 & 35 & Formose & 6254 & 1534 & 55 Kénya & 1464 & 84 & 75 Portugal & 8220 & 1387 & & & \\
\hline 16 & Bulgarte & 13636 & 3636 & 36 & Frence & 39563 & 4140 & 56 Libye & 39754 & 410 & 76 Rhodésie & 2600 & 96 & \multirow{5}{*}{\multicolumn{3}{|c|}{$\begin{array}{l}\text { - si Inga II était ut1lisé } \\
\text { en fait, actuellement, moins } \\
\text { de } 100 \mathrm{khh} \text { par tête }\end{array}$}} \\
\hline 17 & Cameroun & $r 833$ & 202 & 37 & Gabon & 18867 & 755 & 57 Luxembourg & 41390 & 4167 & 77 Roumanio & 8310 & 2816 & & & \\
\hline 18 & Canoda & 40051 & 13360 & 38 & Ghane & 2100 & 381 & 58 Madagascar & 1244 & 30 & 78 Royaume-Un1 & 37600 & 4790 & & & \\
\hline 19 & Centre-Afriq. & 1470 & 294 & 39 & Grèce & 15300 & $2 \pm 01$ & 59 Mala1sie & 5550 & 523 & 79 Sénégal & 2344 & 100 & & & \\
\hline 20 & ent11 & 5285 & 882 & 40 & Guatemala & 4190 & 155 & $60 \mathrm{Ma} 11$ & 616 & 15 & 80 Suède & 46852 & 10800 & & & \\
\hline
\end{tabular}

Figure 1 - Statistiques mondiales 1978 : Consommation (W) en kWh par tête en fonction du PNB (R) en milliers de FF (1978) par tête. Pays riches Autres pays 
les chutes du Népoko qui permettraient de satisfaire très amplement les besoins en énergie de cette région.

L'existence de ces chutes était bien entendu connue de longue date, mais leur éloignement $(90 \mathrm{~km}) \mathrm{du}$ centre de la ville en rendait l'équipement économiquement discutable dans le contexte de la thermie à bas prix.

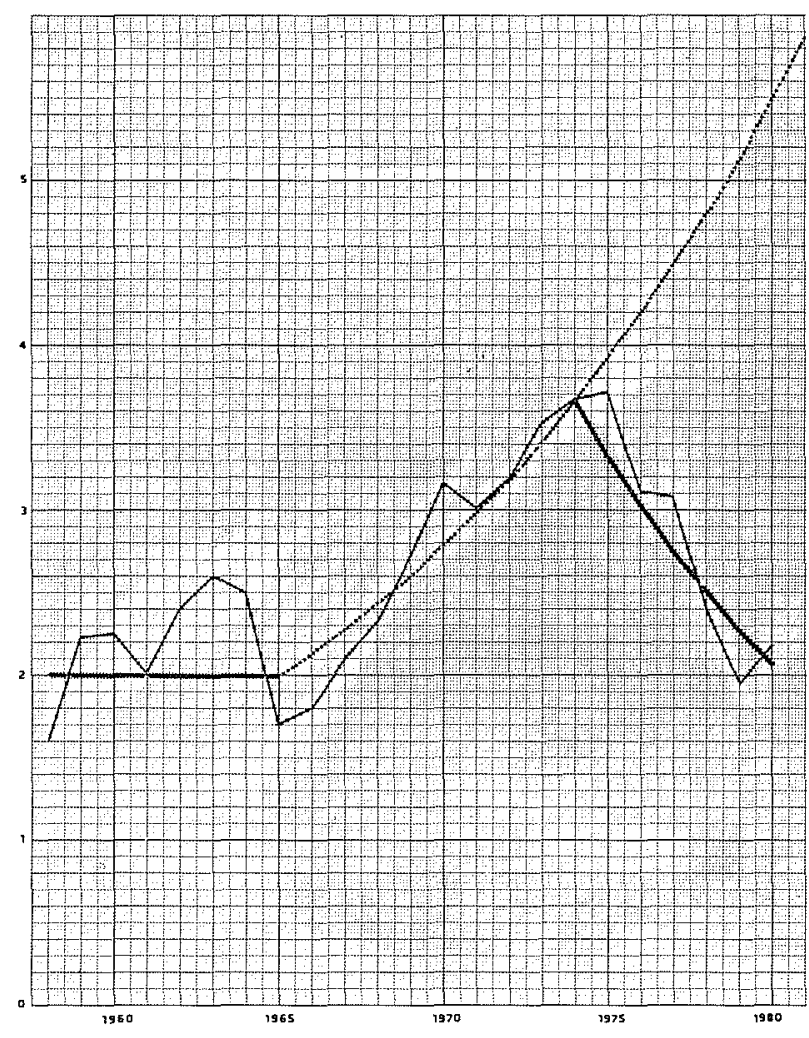

Figure 2 - Production de la Centrale Diesel d'Isiro (Zaïre), en GWh.

\section{Energie-ressource et énergie-besoin}

Certains pays encore peu développés disposent de possibilités hydro-électriques considérables, dépassant largement leurs besoins à court terme. De telles ressources ne peuvent ètre mises à profit que lorsqu'elles sont susceptibles de concurrencer le prix du $\mathrm{kWh}$ (nucléaire) des pays très industrialisés.

Le transport à longues distances de l'électricité pose des problèmes à ce jour insurmontés, et l'utilisation ne peut s'envisager qu'en implantant au voisinage des sites de production des industries fortes consommatrices, à capitaux presque nécessairement étrangers.

A l'inverse, les sites de petites puissances ont évidemment pour vocation la satisfaction des besoins de l'environnement immédiat, et les termes du calcul économique en seront fondamentalement différents.

Les consommations électriques des pays industrialisés se chiffrent en Milliards de $\mathrm{kWh}$, ce qui peut nous faire oublier la valeur de l'unité : pour produire par lui-même un $\mathrm{kWh}$, un homme pesant $75 \mathrm{~kg}$ devrait s'élever de 4900 mètres. Bien peu d'athlètes seraient capables de produire $1 \mathrm{kWh}$ à l'issue d'une épuisante journée de travail, et dans les pays où la force musculaire humaine (et bien souvent féminine...) est encore la source d'énergie la plus répandue, cette référence à la journée de "travailleur de force" prend toute sa signification.

Comparons par exemple ce que représentait un $\mathrm{kWh}$ (aux conditions 1978) pour un Français (11 centimes aux bornes d'un groupe nucléaire) et pour un habitant du Bangladesh ou du Sahel (90 centimes aux bornes d'un petit groupe Diesel).

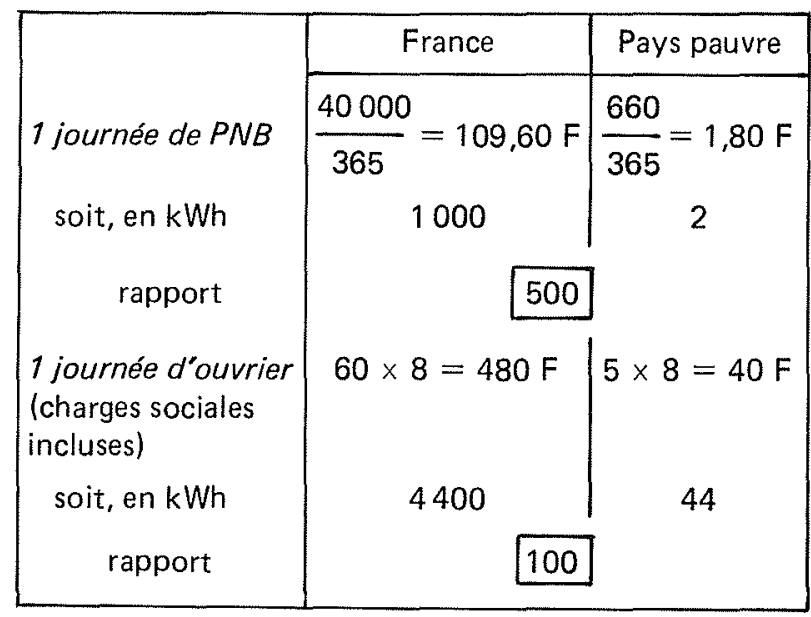

La différence, au premier abord surprenante, entre les deux rapports ainsi dégagés, $s$ 'explique par le fait que dans les pays déshérités les salariés sont des... "privilégiés", en nombre relativement restreint.

Les deux rapports sont l'un et l'autre à considérer : le rapport 100 indique que l'ouvrier d'un pays pauvre peut consacrer 100 fois plus d'heures de travail que le Français à l'équipement d'un $\mathrm{kW}$ de puissance, et le rapport 500 indique que ce serait encore une excellente opération pour l'économie du pays en question.

La conclusion pourrait bien être que les pays en développement plutôt que de rechercher la régulation de fréquence la plus sophistiquée, trouveraient sans doute avantage à construire par eux-mêmes des roues de moulins, comme nous l'avons fait au temps de Vauban... Mais ce serait supprimer le sujet de cette communication.

\section{Le calcul économique}

Certains pays où la consommation électrique est encore très faible peuvent disposer de ressources hydrauliques importantes dont la mise en valeur parait "a priori" très séduisante. Elle devrait permettre en effet d'"exporter" une ressource nationale et de satisfaire, de surcroît, les besoins locaux à des conditions avantageuses.

De telles options peuvent cependant conduire à des erreurs "stratégiques" lourdes de conséquences: on peut citer l'exemple des $1400 \mathrm{MW}$ de l'usine d'Inga II, susceptible de fournir sans doute l'énergie la moins chère du monde, mais pour l'instant inutilisée. On peut citer à l'inverse la réalisation d'Akosombo au Ghana qui mobilise l'essentiel des ressources hydrauliques du pays pour 
alimenter une fonderie d'aluminium à capitaux étrangers : moyennant quoi le pays risque à brève échéance de ne pouvoir faire face à ses propres besoins, ce qui n'empêche pas un pays voisin de nourrir l'ambition de commettre à son tour la même et redoutable erreur.

La mini hydraulique a le grand mérite d'être à l'abri de tels déboires, mais le faible prix de revient au $\mathrm{kW}$ installé de très puissants projets peut lui porter ombrage. Ainsi l'équipement d'une chute voisine d'Isiro, (dont il a été question plus haut) fournirait de l'énergie vraisemblablement 20 fois plus chère que celle d'Inga II, et cet écart énorme a pu suffire à en abandonner l'idée.

C'était peut être lacher la proie pour l'ombre, car mieux vaut encore produire pour soi du $\mathrm{kWh}$ à 80 centimes en substitution du $\mathrm{kWh}$ thermique à 1 franc, que de produire du $\mathrm{kWh}$ à 4 centimes pour le vendre 5 centimes à des étrangers: Charité bien ordonnée commence par soi-même...

Nous n'entrerons pas dans le détail du calcul économique, dont on sait qu'il est conduit en utilisant le principe de l'actualisation.

Les résultats peuvent en être traduits par un graphique qui donnera le prix du $\mathrm{kWh}$ "en moyenne actualisée" en fonction du taux d'actualisation.

Un aménagement thermique, à prédominance de consommations, sera représenté par une droite de faible pente, tandis qu'un aménagement hydraulique à prédominance d'investissements, sera représenté par une droite de forte pente.

Soit (Fig. 3) un aménagement hydraulique $H$ que l'on compare, au niveau de l'étude sommaire, à un Diesel de référence $D_{0}$. Dans le cas de figure choisi, le taux de rentabilité interne de l'aménagement esquissé s'établit à $6,5 \%$, et selon les errements en vigueur il sera très vraisemblablement abandonné.

On installera donc le groupe Diesel et si, pendant ce temps, le prix du diesel-oil a doublé, sa droite représentative s'établira en $D_{1}$, et l'on regrettera certainement de n'avoir pas réalisé $H$.

Ces regrets seront d.autant plus fondés que (s'il était établi de façon sincère) le devis de l'esquisse est affecté d'une marge aléatoire de $\pm 25 \%$ dont on voit à quel point elle influe sur le taux de rentabilité interne. Or, il est tout à fait concevable que des études aussi soignées que celles que l'on entreprend pour des aménagements puissants aient effectivement pour résultat de minorer de $25 \%$ le devis de l'esquisse préliminaire.

Et l'on en arrive ainsi, au problème le plus difficile que posent les petits aménagements: ils sont aussi sensibles que les gros aux différences sur l'investissement, mais le volume global de l'affaire impose de conduire les études dans un tout autre style.

Dans les pays en développement où toute mission de reconnaissance est chère, et où tout est à faire, ce nouveau style d'études reste à trouver, et ne se dégagera pas sans peine.

\section{La collecte des données de base}

L'inventaire systématique des sites favorables est difficilement réalisable, mais ne se présente pas comme



Figure 3 - Comparaison Economique Hydraulique - Thermique "Diesel".

en abscisse : Taux d'actualisation.

en ordonnée : Centimes par kWh "en moyenne actualisée".

de première nécessité : les habitants ont généralement connaissance des chutes et des rapides dans un large périmètre autour de leur localité.

On pourra donc mesurer avec une précision suffisante la hauteur de chute naturelle disponible. Il faudra cependant prendre garde, lorsqu'il s'agit de faibles chutes, que cette hauteur peut être profondément influencée par le débit et l'on trouve fréquemment des sites où une chute intéressante à l'étiage s'efface presque complètement en hautes eaux.

Une bonne connaissance des débits et du régime hydrologique est évidemment nécessaire, et cet impératif est généralement mal perçu. Le plus souvent ce n'est qu'au moment de dresser le projet que l'on se préoccupera de faire les premiers jaugeages, et cette information très insuffisante peut compromettre la rentabilité de l'opération ou la pérennité des ouvrages.

La surestimation du débit d'étiage conduit à des déficits de puissance et la sous-estimation des débits de crues peut être lourde de conséquences et entraîner la ruine des ouvrages: on n'insistera jamais assez sur la nécessité d'entreprendre, suffisamment longtemps à l'avance, l'étude hydrologique qui conditionnera tout le projet.

En ce qui concerne la géologie, on pourra se contenter, le plus souvent, d'une étude très sommaire ; toutefois la reconnaissance de l'épaisseur des terrains de 
couverture doit être conduite avec un maximum de précision car le volume et le coût des ouvrages en fondations prend une importance d'autant plus grande que les parties en élévation sont elles-mêmes plus réduites. S'il est possible de recruter de la main-d'œuvre locale lorsque l'on entreprend l'étude, on trouvera grand avantage à faire exécuter des reconnaissances complètes, par tranchées et par puits : elles peuvent être déterminantes de la conception même des ouvrages, et mettront à l'abri de surprises et d'improvisations lors de leur exécution.

\section{La conduite des études}

Lorsque l'on étudie un aménagement hydroélectrique de forte puissance, la préoccupation majeure est très généralement de concevoir les ouvrages de génie civil qui s'adapteront le mieux aux conditions naturelles du site, et ce n'est qu'après avoir dégagé les lignes essentielles du projet que l'on en viendra à l'étude du matériel proprement dit: prises d'eau, conduites forcées, groupes générateurs.

Lorsqu'il s'agit de petites installations, c'est souvent le processus inverse qu'il conviendra de suivre: les caractéristiques de la chute conduiront au choix d'un certain matériel que l'on s'efforcera ensuite d'inscrire le plus économiquement possible dans le site retenu.

Les petits groupes hydrauliques actuellement proposés sur le marché sont nombreux et variés; par ailleurs, chaque site a sa physionomie propre, et le nombre des combinaisons possibles est considérable. Un essai de classification, même sommaire, déborderait le cadre de cette communication, et cet après-midi même quatre conférenciers traiteront de cette question sous différents aspects.

Le lecteur pourra aussi se reporter utilement à l'étude présentée par Pierre Genin (EDF-DAFECO) au $5^{\mathrm{e}}$ Congrès de l'UPDEA (Kinshasa-février 1976) sur "Les microcentrales hydrauliques - Groupes Bulbes", document disponible au Centre de Documentation EDF 2, rue Louis Murat 75008 Paris ;

Les conclusions de cette étude sont que les conditions favorables à la réalisation d'une micro-centrale hydraulique sont :

- un site adapté (génie civil réduit);

- une installation robuste et simplifiée au maximum; - une ligne de transport d'énergie courte $(10 \mathrm{~km}$ à $20 \mathrm{~km}$ maximum);

- une bonne utilisation annuelle (3000 heures au moins) de la puissance installée.

\section{La réalisation}

Dans le domaine de l'hydroélectricité, l'effet de taille est particulièrement sensible et les petites installations se révéleront généralement plus chères, au $\mathrm{kW}$ installé, que les aménagements de forte puissance.

Il vient s'y ajouter que certains frais fixes (ouverture d'une piste d'accès au site par exemple) ainsi que les frais de direction et de contrôle des travaux représenteront un pourcentage non négligeable de l'investissement.
En conséquence, il convient de privilégier systématiquement les solutions qui permettront une exécution rapide, d'étudier avec soin le planning d'ensemble, et de veiller fermement au respect des délais.

Chaque pays, chaque région a ses ressources spécifiques tant en ce qui concerne la disponibilité et la qualification de la main-d'œuvre qu'en possibilités de recours aux matériaux locaux.

Ces éléments doivent être pris en compte dès l'origine de l'étude et peuvent être déterminants dans le choix de telle ou telle solution, tout particulièrement dans le domaine du génie civil.

La Chine offre sans doute le plus important exemple de recours aux ressources et aux compétences strictement locales pour de telles réalisations: les trois quarts de la production électrique destinée à satisfaire les besoins des communes rurales proviennent de microcentrales au nombre de 60000 , d'une puissance moyenne de $40 \mathrm{~kW}$.

Turbines, générateurs, pièces de chaudronnerie, et matériels accessoires sont produits dans des ateliers régionaux de façon standardisée, et le génie civil fait le plus large appel aux matériaux traditionnels : bois, terre, ou maçonnerie.

La conduite de ces petites unités est directement prise en charge par la collectivité utilisatrice;l'entretien et la maintenance sont assurés par des "équipes volantes" au plan régional.

Par ce choix politique qui s'appuie sur l'ingéniosité de ses citoyens, la Chine fait figure, en ce domaine, de pays particulièrement développé: les pays en développement pourront s'inspirer de cet exemple pour ce qui concerne l'exploitation et l'entretien, mais suivront difficilement ce modèle pour ce qui est des réalisations elles-mêmes.

C'est en effet le très grand nombre de micro-projets à réaliser qui a permis de concevoir et de mener à bien un tel plan d'équipement, en surmontant vraisemblablement un certain nombre de déboires ou d'échecs, à ses débuts.

Pour les pays qui ne disposent ni d'un pareil champ d'action, ni peut être d'un même efficacité individuelle, la mini-hydroélectricité peut être le fruit d'une coopération particulièrement intéressante avec les pays industrialisés possédant une longue expérience technique en la matière : on peut rappeler à ce sujet qu'en France par exemple un millier d'installations de moins de $1 \mathrm{MW}$ sont en service et que l'on continue d'en équiper.

Dans cette cuvre de coopération, l'un des partenaires fait apport de son expérience, de sa technologie, et de son outillage industriel; l'autre partenaire dispose généralement d'une main d'œuvre abondante, et parfois de traditions intéressantes dans l'art de construire en utilisant les ressources locales.

Dans ces conditions, la répartition des responsabilités et des tâches la plus souhaitable paraît être la suivante: - le pays industrialisé dresse le projet, puis foumit des matériels qui doivent être compacts, robustes, fiables, et par conséquent de conception et de réalisation très voisines de celles qu'il a déjà mises au point pour ses besoins propres.

On attachera cependant une particulière importance à la rapidité et à la facilité des assemblages ou montages 
à réaliser sur le site même: par raison d'économie, l'intervention des monteurs spécialistes doit être aussi brève que possible, et les futurs utilisateurs doivent être associés à ces opérations pour être en mesure de les. reprendre en cours d'exploitation.

- dans la mesure où son niveau de technicité le lui permet, le pays en voie de développement prend en charge la réalisation des ouvrages de génie civil, en faisant le plus large appel à ses ressources et à ses traditions : ainsi s'enrichira-t-il d'une expérience qui devrait le conduire assez rapidement à une réelle indépendance en la matière.

- si le niveau de technicité est insuffisant, il faudra faire appel à un encadrement étranger pour l'exécution des travaux de génie civil. Cette intervention aura bien entendu ses répercussions sur le coût des ouvrages, et les techniciens expatriés devraient être suffisamment compétents sur le plan de la formation professionnelle pour que leur rôle devienne de moins en moins nécessaire.

\section{Conclusion}

La nécessité d'un "transfert du capital technique" qui vient d'être abordée est l'évidente condition du cheminement sur la voie du "développement".
Il est reconnu que les pays en développement disposent d'une main-d'œuvre abondante et sous-employée, mais non qualifiée, et d'autre part d'une élite formée dans les universités nationales ou étrangères qui n'a souvent que peu d'attrait pour le travail "sur le tas". La catégorie intermédiaire : ouvriers spécialisés et maitrise, est presque inexistante et cette lacune est un obstacle majeur à de réels progrès.

Par rapport aux autres produits de la civilisation industrielle, l'énergie hydraulique est d'un abord relativement facile. Elle fait appel pour l'essentiel à des notions intuitives, mais concrètes : pressions, hauteurs, débits sont des grandeurs visibles dont le sens est immédiat.

Lorsqu'il s'agit de petites installations, chaque participant est en mesure de saisir l'ensemble du sujet et d'apprécier la valeur de sa contribution personnelle à l'ouvrage entrepris.

Ce sont de remarquables conditions pour la formation de catégories professionnelles dont les pays en développement ont le plus grand besoin et la mini-hydraulique - en dehors de ses aspects purement économiquesoffre à la coopération internationale l'une des voies les plus sûres pour aborder ce problème essentiel.

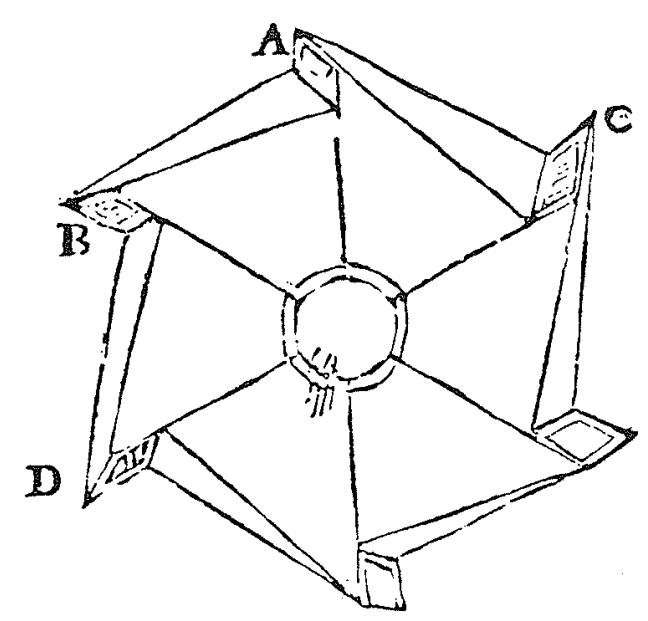

